CORRECTION

\title{
Correction: Dose-Related Effects of Adjunctive Ketamine in Taiwanese Patients with Treatment-Resistant Depression
}

Tung-Ping Su ${ }^{1,2,3,4}$, Mu-Hong Chen ${ }^{2,4}$, Cheng-Ta Li ${ }^{1,2,4}$, Wei-Chen Lin $^{2,4}$, Chen-Jee Hong ${ }^{1,2,4}$, Ralitza Gueorguieva ${ }^{5,6}$, Pei-Chi Tu ${ }^{1,2,3}$, Ya-Mei Bai ${ }^{1,2}$, Chih-Ming Cheng ${ }^{2}$ and John H. Krystal ${ }^{6,7,8,9}$

Neuropsychopharmacology (2019) 44:655-656; https://doi.org/10.1038/s41386-018-0253-z

Correction to: Neuropsychopharmacology (2017), https://doi.org/ 10.1038/npp.2017.94

Following the publication of this article the authors noted an error in Fig. 5. The units of blood concentration for ketamine and norketamine should be $\mathrm{ng} / \mathrm{mL}$, as per the assay details in Materials and methods section, and not $\mathrm{nM} / \mathrm{ml}$ as presented in the figure. A revised Fig. 5 is in the correction article. The authors apologize for any inconvenience caused.

\footnotetext{
'Division of Psychiatry, Faculty of Medicine, National Yang-Ming University, Taipei, Taiwan; ${ }^{2}$ Department of Psychiatry, Taipei Veterans General Hospital, Taipei, Taiwan; ${ }^{3}$ Department of Medical Research, Taipei Veterans General Hospital, Taipei, Taiwan; ${ }^{4}$ Institute of Brain Science, National Yang-Ming University, Taipei, Taiwan; ${ }^{5}$ Department of

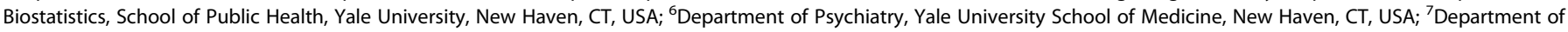
Neuroscience, Yale University School of Medicine, New Haven, CT, USA; ${ }^{8}$ Yale-New Haven Hospital, New Haven, CT, USA and ${ }^{9}$ VA National Center for PTSD, VA Connecticut Healthcare System, West Haven, CT, USA

Correspondence: T-P. Su (tomsu0402@gmail.com) or John H. Krystal (john.krystal@yale.edu)
}

Published online: 28 November 2018 
Correction: Dose-Related Effects of Adjunctive Ketamine in Taiwanese...

T-P Si et al.

656

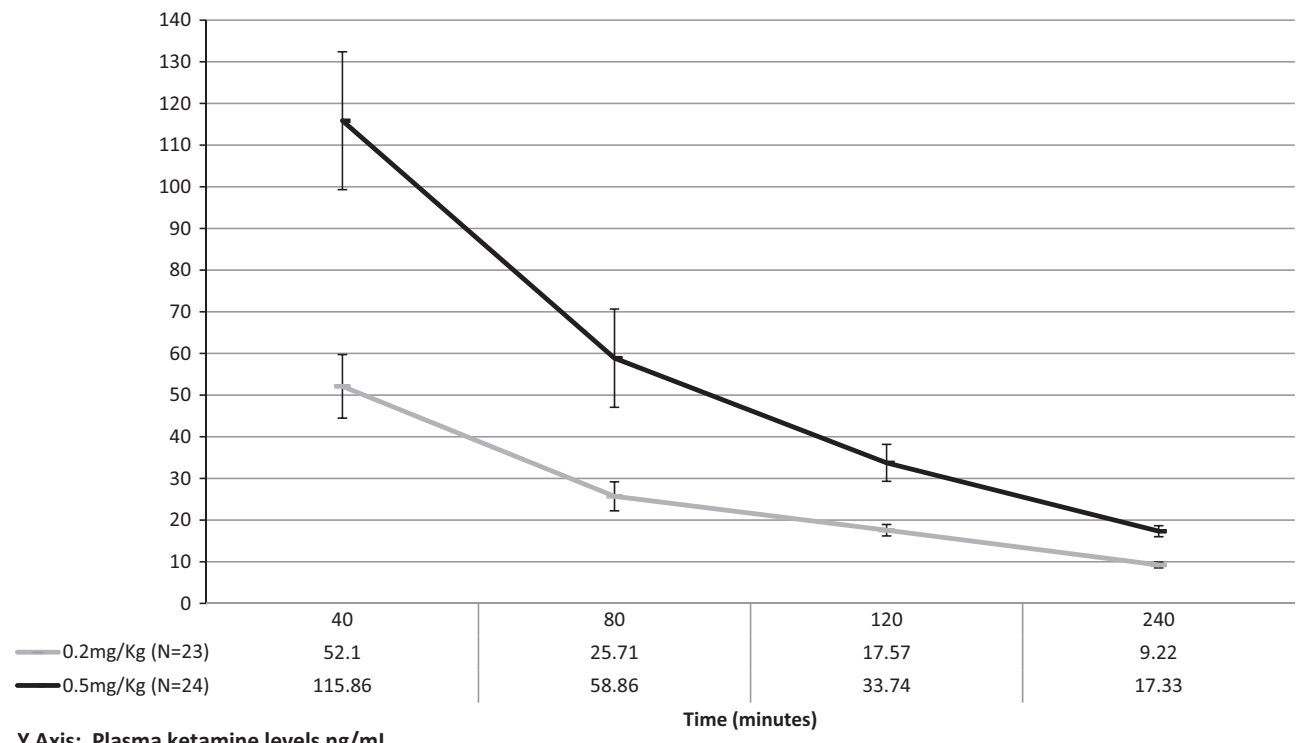

Y Axis: Plasma etamine levels $\mathrm{ng} / \mathrm{mL}$

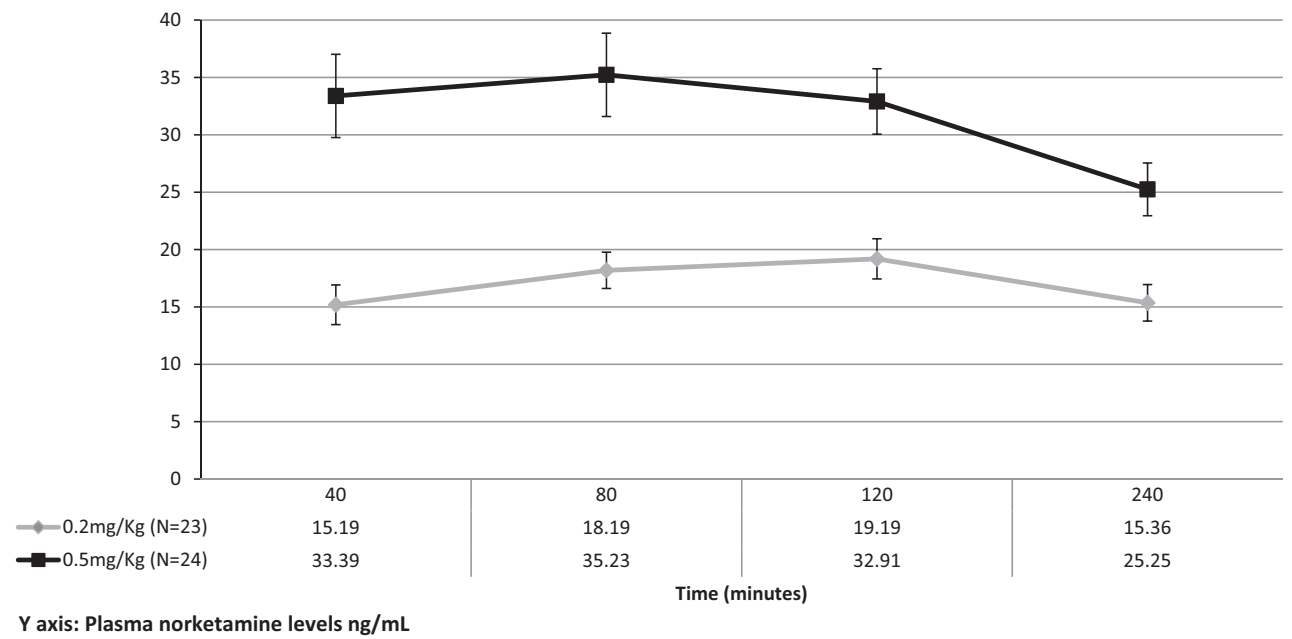

Fig. 5

SPRINGER NATURE

Neuropsychopharmacology (2019) 44:655-656 